\title{
1 Single-vessel cerebral blood flow fMRI to map blood velocity by phase-contrast 2 imaging
}

4 Xuming Chen ${ }^{1,2}$, Yuanyuan Jiang ${ }^{3}$, Sangcheon Choi ${ }^{1,4}$, Rolf Pohmann ${ }^{1}$, 5 Klaus Scheffler ${ }^{1,5}$, David Kleinfeld ${ }^{6,7}$ and Xin $\mathrm{Yu}^{1,3,8,{ }^{*}}$

$6 \quad{ }^{1}$ High-Field Magnetic Resonance, Max Planck Institute for Biological Cybernetics, 72076

7 Tübingen, Germany.

$8{ }^{2}$ Department of Neurology, Wuhan University, Renmin Hospital, Wuhan 430060, China.

$9 \quad{ }^{3}$ Athinoula A. Martinos Center for Biomedical Imaging, Massachusetts General Hospital and

10 Harvard Medical School, Charlestown 02129 MA, USA.

$11{ }^{4}$ Graduate Training Centre of Neuroscience, International Max Planck Research School, 12 University of Tübingen, 72074 Tübingen, Germany.

13 5Department for Biomedical Magnetic Resonance, University of Tübingen, Tübingen, Germany.

$14{ }^{6}$ Department of Physics, University of California at San Diego, La Jolla, CA, 92093 USA.

$15{ }^{7}$ Section of Neurobiology, University of California at San Diego, La Jolla, CA, 92093 USA.

27 'Lead Contact:

28 Address: Max-Planck-Ring. 11, 72076, Tübingen, Germany

29 Phone: $+497071601-740$

30 Fax: $+497071601-701$

$31{ }^{*}$ Correspondence: xin.yu@tuebingen.mpg.de; xyu9@mgh.harvard.edu 


\section{Abstract}

34 Current approaches to high-field fMRI provide two means to map hemodynamics at the

35 level of single vessels in the brain. One is through changes in deoxyhemoglobin in

36 venules, i.e., blood oxygenation level-dependent (BOLD) fMRI, while the second is

37 through changes in arteriole diameter, i.e., cerebral blood volume (CBV) fMRI. Here we

38 introduce cerebral blood flow (CBF)-fMRI, which uses high-resolution phase-contrast MRI

39 to form velocity measurements of flow and demonstrate CBF-fMRI in single penetrating

40 microvessels across rat parietal cortex. In contrast to the venule-dominated BOLD and

41 arteriole-dominated CBV $\mathrm{fMRI}$ signal, the phase-contrast -based CBF signal changes are

42 highly comparable from both arterioles and venules. Thus, we have developed a single-

43 vessel fMRI platform to map the BOLD, CBV, and CBF from penetrating microvessels

44 throughout the cortex. This high-resolution single-vessel fMRI mapping scheme not only

45 enables the vessel-specific hemodynamic mapping in diseased animal models but also

46 presents a translational potential to map vascular dementia in diseased or injured human

47 brains with ultra-high field fMRI.

48

49

50

51

52

53 


\section{Summary}

56 We established a high-resolution PC-based single-vessel velocity mapping method using

57 the high field MRI. This PC-based micro-vessel velocity measurement enables the

58 development of the single-vessel CBF-fMRI method. In particular, in contrast to the

59 arteriole-dominated CBV and venule-dominated BOLD responses, the CBF-fMRI shows

60 similar velocity changes in penetrating arterioles and venules in activated brain regions.

61 Thus, we have built a noninvasive single-vessel fMRI mapping scheme for BOLD, CBV,

62 and CBF hemodynamic parameter measurements in animals.

63

64

65 Keywords: Phase contrast, cerebral blood flow, velocity, single vessel, fMRI, blood 


\section{Introduction}

68 Cerebral blood flow (CBF) is a key readout of neuronal processing and viability in normal

69 and diseased brain states ${ }^{1}$. Changes in CBF may be monitored directly within individual

70 blood vessels through the use of optical-based particle tracking techniques ${ }^{2}$. A variety of

71 imaging methods have been developed to measure CBF across multiple spatial scales

72 from capillary beds to the vascular network in animal brains, including multi-photon

73 microscopy $^{3}$, near-infrared spectroscopy $(\mathrm{NIRS})^{4}$, optical coherence tomography ${ }^{5}$,

74 optoacoustic imaging ${ }^{6}$, or laser doppler and speckle imaging ${ }^{7,8}$. In particular, the doppler-

75 based functional ultrasound imaging method provides a unique advantage to detect the

76 CBF in the brain with a high spatiotemporal resolution, which can be readily applied for

77 awake animal imaging ${ }^{9-11}$. However, these methods share a common barrier that the

78 spectrum-specific signal transmission cannot effectively pass the skull of animals without

79 significant loss of the signal-to-noise ratio (SNR). Typically, a craniotomy or procedure to

80 thin the skull is needed to detect the hemodynamic signal ${ }^{2}$. While current techniques

81 support transcranial imaging into the superficial layers of the cortex, only functional MRI

82 (fMRI) provides a noninvasive approach for measuring hemodynamic signals throughout

83 the brain.

$84 \quad$ Changes in CBF may be detected by $\mathrm{fMRI}$ based on arterial spin labeling (ASL),

85 in which water protons in a major upstream vessel are spin-polarized with an external

86 field ${ }^{12-14}$. Two other fMRI-based techniques provide indirect information about changes in

87 CBF. Blood oxygenation level-dependent (BOLD) $\mathrm{fMRI}$ is used to determine changes in

88 the ratio of deoxy- to oxyhemoglobin in the blood and is a measure of changes in brain

89 metabolism ${ }^{12,15,16}$. Cerebral blood volume (CBV) fMRI is used to measure changes in 
90 blood volume, i.e., essentially changes the diameter of arterioles, based on the use of

91 exogenous or endogenous contrast agents to differentiate blood from brain tissue ${ }^{12,17 .}$

Phase-contrast (PC) MRI relies on gradient-oriented dephasing of magnetized

93 protons to map the velocity, i.e., direction and speed, of blood flow ${ }^{18,19}$. The ASL-based

94 CBF fMRI technique detects local changes in the flow of blood through brain tissue but

95 does not show orientation-specific information related to the alignment of vessels ${ }^{20}$. Past

96 works with 7 T MR scanning showed that PC-MRI can be used to measure flow in the

97 perforating arteries through the while matter or the lenticulostriate arteries in the basal

98 ganglia of human brains ${ }^{21-24}$. However, the SNR was insufficient in these prior studies to

99 map changes in flow, and thus changes in CBF.

$100 \quad$ Here, we report on a PC-MRI method to detect the vessel-specific changes in

101 blood velocity in single trials. Compared with past implementations of PC-MRI ${ }^{21,25-28}$, we

102 have implemented a small surface radio frequency (RF) coil with the high field MRI, i.e.,

103 14.1 T for improved SNR. This further allows us to map the BOLD- and CBV-fMRI from

104 individual penetrating venules and arterioles, which span 20 to $70 \mu \mathrm{m}$ diameter, with high

105 spatial resolution $29-31$.

106 Results

107 Phantom validation of high-resolution PC-based flow velocity measurement

108 For calibration, we constructed an in vitro capillary tubing circulatory system to mimic

109 penetrating vessels, with flow rates from 1 to $10 \mathrm{~mm} / \mathrm{s}$ (Figure 1A). A 2D PC-MRI slice is

110 aligned perpendicular to the capillary tubing (Figure 1A, B) and provides a voxel-specific

111 measurement of the flow velocity through two tubes with the upward flow (positive sign,

112 bright dots in Figure 1B) and two tubes with the downward flow (negative sign, dark dots

113 in Figure 1B), as well as a control tube. We observe a monotonic and near-linear relation 
114 between the velocity measured by PC-MRI and the true velocity: $\mathrm{V}_{\text {meas }}=$

$115(0.67 \pm 0.01) v_{\text {pump }}+(0.02 \pm 0.11) \mathrm{mm} / \mathrm{s}$ at echo time $(\mathrm{TE})=5.0 \mathrm{~ms}$ (Figure 1C). The

116 small offset could be caused by eddy current effects and other gradient-related scaling

117 errors of the PC-MRI sequence ${ }^{32-34}$. We further observe that the measured velocities are 118 relatively insensitive to the value of TE (Figure 1C).

We implemented the high-resolution PC-MRI for in vivo measurement of blood flow from individual penetrating arterioles and venules through the infragranular cortex, i.e.,

121 layer $\mathrm{V}$, of the anesthetized rats with 14.1 T MR scanning. To improve the SNR of PC-

$122 \mathrm{MRI}$ images as well as multi-gradient echo (MGE) images used for arteriole-venule (A-V)

123 mapping 29,31 , a surface RF transceiver coil with $6 \mathrm{~mm}$ diameter was constructed and

124 attached to the rat skull (Supplemental Figure 1). This was essential for the high-

125 resolution mapping with a fast sampling rate of the single-vessel flow velocity over a

126 complete hemisphere of the rat brain (Figure 2A and Supplemental Figure 1E).

127 In vivo PC-based flow velocity mapping of penetrating microvessels

128 We first acquired the single-vessel A-V map by aligning a $500 \mu \mathrm{m}$ thick 2D MRI slice 129 perpendicular to the penetrating vessels through layer $\mathrm{V}$ of one hemisphere (Figure 2A,

130 B). We designed the pulse sequence for PC-MRI to achieve the same slice geometry of

131 the $\mathrm{A}-\mathrm{V}$ map so that the CBF deduced from PC-MRI signals could be overlaid with

132 individual penetrating arterioles and venules in the single-vessel flow velocity map

133 (Figure 2A, C). The arteriole blood flows down into the cortex while the venule blood

134 flows upward, which determines the sign of the flow velocity. Vessel-specific velocities

135 were plotted as a function of the normalized signal intensity in the $A-V$ map and 136 corroborated our ability to determine flow velocity specific to arterioles and venules

137 (Figure 2C, D). The measured flow velocities range from 1 to $10 \mathrm{~mm} / \mathrm{s}$, as previously 
138 measured with optical methods ${ }^{35}$. To probe the reliability of the single-vessel MR-based

139 flow velocity method, we compared the velocities detected by PC-MRI methods with

140 different TEs and flip angles (FAs) and observed comparable results across a range of

141 parameters (Supplemental Figure 2). All told, these data demonstrate the feasibility of

142 in vivo single-vessel blood velocity mapping with the PC-based MRI method.

143 PC-based CBF-fMRI from individual arterioles and venules

144 We contrasted the complementary capabilities of PC-based CBF-fMRI against the signals

145 observed with the balanced steady-state free precession (bSSFP)-based single- vessel

146 BOLD- and CBV-fMRI mapping method ${ }^{29}$ (Figure 3). We first created an A-V map

147 through the deep layers of the forepaw region of the primary somatosensory cortex

148 (Figure 3Ai), followed by 2D-bSSFP to detect stimulus-induced changes in the single-

149 vessel BOLD-fMRI signal (Figure 3Aii). We next performed single-vessel PC-MRI flow

150 velocity measurements with $100 \times 100 \mu \mathrm{m}^{2}$ in-plane resolution, a sampling rate of $4 \mathrm{~s}$

151 repetition time (TR) per image, and the same geometry as the 2D-bSSFP method to

152 measure baseline flow in penetrating arterioles and venules (Figure 3Aiii). Changes in

153 CBF upon stimulation overlapped with individual penetrating vessels in the $A-V$ map

154 (Figure 3Aiv). Lastly, we performed 2D-bSSFP for single-vessel CBV-fMRI mapping by

155 intravenous injection of iron particles into the blood in the same rats (Figure $\mathbf{3 A v}$ ). The

156 BOLD-fMRI signal is primarily detected from individual penetrating venules while the

157 CBV-weighted signal is mainly located at the individual penetrating arterioles (dark dots

158 in Figure 3Aii with bright dots in Figure 3Av). In contrast, the CBF-fMRI signal is

159 observed in both penetrating arterioles and venules (Figure 3Aiv). 
The stimulus-evoked responses of all three fMRI signals were studied with an on/off block design (Figures 3B-E). Group analysis shows that the positive BOLD signal

162 from venule voxels is significantly higher than the arteriole-specific BOLD signal

163 (Figure 3C). In contrast, the arteriole dilation leads to an earlier CBV-weighted negative

$164 \mathrm{fMRl}$ signal, which is much stronger and faster than the signal from passive venule-

165 dilation (Figure 3C), as expected ${ }^{36,37 . ~ G r o u p ~ a n a l y s i s ~ s h o w s ~ t h e ~ s i m i l a r ~ t e m p o r a l ~}$ 166 dynamics of CBF changes appearing as a ramp in both arterioles and venules

167 (Figure 3E). The CBF-fMRI signal appears as the integral of the stimulus, i.e., a triangular 168 ramp (Figure 3E), compared to the saturation-like BOLD and CBV responses 169 (Figure 3C). The voxel-wise hemodynamic changes of BOLD, CBV, and CBF are $170 \quad$ illustrated in Supplementary Figure 3 and movie 1.

\section{Discussion}

172 Despite the existing tools developed for CBF measurement in both animal and human

173 brains, it remains challenging to detect the flow dynamics of intracortical micro-vessels

174 non-invasively. Here, we not only optimized the PC-MRI to map the vectorized single-

175 vessel flow velocity of penetrating arterioles and venules but also developed the single-

176 vessel CBF-fMRI based on the direct flow velocity measurement in rat brains. By

177 combining with previously established single-vessel BOLD- and CBV-fMRI methods, the

178 PC-based single-vessel CBF-fMRI method complements the scheme to map the vessel-

179 specific hemodynamic responses with high-resolution fMRI.

In contrast to the conventional ASL methods, PC-based MRI mapping allows

181 arterioles and venules to be distinguished for simultaneous velocity measurements 182 through a 2D plane. Also, ASL has less vascular specificity because water exchange 
183 through the blood-brain barrier of capillary beds increases the weighting of the ASL-based

184 flow signal for parenchyma voxels ${ }^{38,39}$. Furthermore, there is significant variability in the

185 transit time to flow from arterioles to venules through the capillary bed ${ }^{40}$, which

186 complicates the distinction of arterioles and venules by simple ASL-based CBF mapping.

187 We detected the velocity from penetrating microvessels in the deep cortical layers with

188 PC-MRI, showing velocity values from 1 to $5 \mathrm{~mm} / \mathrm{s}$ (Fig 2). Single-digit velocity (mm/s) of

189 red blood cells from cortical surface microvessels has been detected in the anesthetized

190 rats using two-photon microscopy ${ }^{2,35}$. It is noteworthy that the PC-based vessel velocity

191 measurement is based on measuring water protons in blood but not limited to the flow of

192 red blood cells. Still, the PC-based velocity from microvessels matches well with the

193 previous optical measurement. We conclude that high-resolution PC-MRI is ideal for

194 noninvasive single-vessel CBF-fMRI mapping.

A remaining complication with $\mathrm{PC}-\mathrm{MRI}$ mapping is the presence of small offsets in velocity from the phantom capillary tubing with circulating flow under different conditions.

197 The phase-dependent velocity encoding depends on the quality of the magnetic field 198 gradients, and mismatched eddy currents of multiple gradients with opposite polarities, 199 as well as the nonlinear and distorted gradient fields, could contribute to distortions in 200 gradients ${ }^{32-34}$. In particular, the high-resolution PC-MRI method is a high-duty cycle 201 sequence and slight heating of the gradient coil during scanning may alter the gradient 202 performance, consistent with the baseline-drift of the CBF-fMRI signal in the first 5 203 minutes of scanning (Figure 3D). Nevertheless, it should be noted that the percentage 204 velocity changes from individual arterioles and venules can be readily detected with the 205 PC-based CBF-fMRI measurement regardless of the gradient-heating related baseline 
207 the limited spatial resolution of the PC-MRI images compared to the diameters of small

208 vessels $^{27}$, although corrections are possible ${ }^{21,28}$. Despite the potential partial volume

209 contribution to the single-vessel BOLD, CBV, and CBF-fMRI, the vessel-specific mapping

210 scheme presents a translational potential to identify vascular dementia in diseased or

211 injured brains with ultra-high field fMRI.

\section{ACKNOWLEDGMENTS}

213 We thank Dr. E. Weiler, Ms. H. Schulz, and Ms. S. Fischer for animal/lab maintenance and

214 support, Dr. K. Buckenmaier and Dr. N. Avdievitch for technical support, and the Analysis of

215 Functional Neurolmages (AFNI) team for their software support. This research was supported by

216 NIH BRAIN grants R01 1NS113278 and R01 MH111438, NIH NINDS grant R35 NS097265, NIH

217 instrument grant S10 RR023009 to the Massachusetts General Hospital/Harvard-MIT Program in

218 Health Sciences and Technology Martinos Center, Deutsche Forschungsgemeinschaft (DFG,

219 Germany Research Foundation) grant YU 215/3-1, SCHE 658/15, SCHE 658/12, the 220 Bundesministerium fuer bildung und forschung (BMBF, Federal Ministry of Education and 221 Research) grant 01GQ1702, and the Chinese Scholarship Council for the doctoral support of 222 X. Chen.

\section{AUTHOR CONTRIBUTIONS}

224 X.Y. and D.K. initiated the concept; X.Y. designed the research; X.C. and X.Y. performed animal 225 experiments; X.C., Y.J. performed data analysis; P.R., K.S., S.C. provided technical support; X.Y., 226 D.K., X.C., Y.J. wrote the paper. 


\section{Methods}

\section{Design of a phantom capillary tubing flow system}

231 In order to validate the PC-MRI sequence, a plastic circulatory flow phantom composed 232 of the capillary tubing (PE-10, Instech Laboratories, inner diameter $210 \mu \mathrm{m}$ ) was 233 constructed to mimic the geometries of cortical blood vessels (Figure 1A). The capillary 234 tube was connected to a programmable syringe infusion/withdraw pump (Pump Elite 11,

235 Harvard Apparatus) with an infusion rate of $0.25,0.5,1.0,1.5,2.0 \mathrm{ml} / \mathrm{h}$, which were 236 transferred to the flow velocity of the capillary tubing as shown in Figure 1. The flowing 237 medium is a manganese solution ( $50 \mathrm{mM} \mathrm{MnCl}_{2}$, Sigma-Aldrich). The phantom tube was 238 cast with Fomblin (Sigma-Aldrich) to avoid the potential air interface artifacts.

\section{Animal preparation for fMRI}

240 All surgical and experimental procedures were approved by the local authorities

241 (Regierungspraesidium, Tübingen Referat 35, Veterinärwesen, Leiter Dr. Maas) and 242 were in full compliance with the guidelines of the European Community (EUVD $24386 / 609 / E E C)$ for the care and use of laboratory animals. The experimental animals were 244 Sprague-Dawley male rats, $\sim 250 \mathrm{~g}$, provided by the Charles River Laboratories in 245 Germany. Fifteen rats were used in all experiments (the evoked bSSFP-BOLD/CBV and 246 PC-MRI signals were acquired from five of these fifteen rats).

247 Detailed descriptions of the surgery are given in previous publications ${ }^{29,}{ }^{30}$. Briefly, 248 rats were first anesthetized with isoflurane (5\% induction, 1 2\% maintenance), each rat 249 was orally intubated with a mechanical ventilator (SAR-830, CWE). The femoral artery 250 and vein were catheterized with plastic catheters (PE-50, Instech Laboratories) to monitor 251 the arterial blood gas, administrate drugs, and constantly measure the blood pressure. 
252 After catheterization, rats were secured in a stereotaxic apparatus, a custom-made RF

253 coil was fixed above the skull with cyanoacrylate glue (454, Loctite). After surgery,

254 isoflurane was switched off and a bolus of $\alpha$-chloralose (80 mg/kg, Sigma-Aldrich) was

255 intravenously injected. A mixture of $\alpha$-chloralose $(26.5 \mathrm{mg} / \mathrm{kg} / \mathrm{h})$ and the muscle relaxant

256 (pancuronium bromide, $2 \mathrm{mg} / \mathrm{kg} / \mathrm{h}$ ) was continuously infused to maintain the anesthesia

257 and minimize the motion artifacts. Throughout the whole experiment, the rectal

258 temperature of rats was maintained at $37^{\circ} \mathrm{C}$ by using a feedback heating system. All

259 relevant physiological parameters were constantly monitored during imaging, including

260 heart rate, rectal temperature, arterial blood pressure, the pressure of the tidal ventilation,

261 and end-tidal $\mathrm{CO}_{2}$. Arterial blood gases were checked to guide the physiological status

262 adjustments by changing the respiratory volume or administering sodium bicarbonate

263 (NaBic $8.4 \%$, Braun) to maintain normal pH levels. Dextran-coated iron oxide particles

264 (15 $20 \mathrm{mg}$ of Fe/kg, BioPAL, MA) were intravenously injected for CBV-weighted signal 265 acquisition.

266 fMRI setup

267 All images were acquired with a 14.1 T, $26 \mathrm{~cm}$ horizontal bore magnet (Magnex Scientific)

268 interfaced through the Bruker Advance III console (Bruker Corporation). The scanner is 269 equipped with a $12 \mathrm{~cm}$ magnet gradient set capable of providing a strength of $100 \mathrm{G} / \mathrm{cm}$

270 and a $150 \mu$ s rise time (Resonance Research Inc.). A custom-made transceiver coil with

271 an internal diameter of $6 \mathrm{~mm}$ was used for $\mathrm{fMRI}$ images acquisition. For the electrical

272 stimulation, two custom-made needle electrodes were placed on the forepaw area of the

273 rats to deliver the electrical pulse sequences (330 $\mu$ s duration at $1.0 \sim 2.0 \mathrm{~mA}$. The pulses

274 repeated at $3 \mathrm{~Hz}$ for $10 \mathrm{~s}$ ) by using a stimulus isolator (A365, WPI). The stimulation

275 duration and frequency were triggered directly through the MRI scanner which controlled 
276 by Master-9 A.M.P.I system (Jerusalem, Israel). The triggering pulses from the MRI

277 scanner were also recorded by the Biopac system (MP150, Biopac Systems, USA).

278 Single-vessel multi-gradient echo (MGE) imaging

279 To anatomically map the individual arterioles and venules penetrating the deep cortical

280 layers of the somatosensory cortex, a 2D MGE sequence was applied with the following 281 parameters: $\mathrm{TR}=50 \mathrm{~ms} ; \mathrm{TE}=2.5,5.0,7.5,10.0,12.5$ and $15.0 \mathrm{~ms}$; flip angle $(\mathrm{FA})=55^{\circ}$; 282 matrix $=192 \times 192$; in-plane resolution $=50 \times 50 \mu \mathrm{m}^{2}$; slice thickness $=500 \mu \mathrm{m}$. The A-V 283 map was made by averaging the MGE images from the second TE echo to the fifth TE 284 echo. In the A-V map, the arteriole voxels show bright (red marks) due to the in-flow effect 285 and venule voxels show as dark dots (blue marks) because of the fast $\mathrm{T}_{2}{ }^{*}$ decay 286 (Figure 2B).

287 Balanced steady-state free precession (bSSFP) BOLD- and CBV-fMRI

288 The bSSFP sequence was applied to acquire the evoked BOLD signals by using the 289 following parameters: $\mathrm{TR}=15.6 \mathrm{~ms} ; \mathrm{TE}=7.8 \mathrm{~ms}$; flip angle $=15^{\circ}$; matrix $=96 \times 96$; FOV $290=9.6 \times 9.6 \mathrm{~mm}$; in-plane resolution $=100 \times 100 \mu \mathrm{m}^{2}$; slice thickness $=500 \mu \mathrm{m}$. For the 291 bSSFP CBV-fMRI, the parameters were adjusted with $\mathrm{TR}=10.4 \mathrm{~ms}$ and TE $=5.2 \mathrm{~ms}$.

292 The total TR to acquire each image is $1 \mathrm{~s}$. To reach the steady-state, 300 dummy scans 293 were used, followed by 25 pre-stimulation scans, one scan during stimulation, and 44 294 post-stimulation scans with 10 epochs for each trial. The fMRI stimuli block design of each 295 trial consisted of $10 \mathrm{~s}$ stimulation and $35 \mathrm{~s}$ inter-stimulus interval. The total acquisition 296 duration of each trial was 7 min $55 \mathrm{~s}$. CBV-weighted fMRI signals were acquired after 297 intravenous injection of dextran-coated iron oxide particles (15 $20 \mathrm{mg}$ of Fe/kg, BioPAL, 298 MA). 


\section{Phase Contrast (PC)-MRI}

300 To measure the flow velocity of individual arterioles and venules, the PC-MRI sequence was applied with the following parameters. For the in vitro phantom measurement: $\mathrm{TR}=$ $15.6 \mathrm{~ms} ; \mathrm{TE}=4.2,4.5,5.0,6.0 \mathrm{~ms}$; flip angle $=25^{\circ} ; \mathrm{FOV}=6.4 \times 6.4 \mathrm{~mm}$; matrix $=$ $128 \times 128$; in-plane resolution $=50 \times 50 \mu \mathrm{m}^{2}$; slice thickness $=500 \mu \mathrm{m}$; maximum velocity

304 (Venc) $=1.56$ or $0.66 \mathrm{~cm} / \mathrm{s}$ (based on the flow values); number of averages $=172$. The 305 total acquisition time was $11 \mathrm{~min} 28 \mathrm{~s}$. For the in vivo measurements: TR $=15.6 \mathrm{~ms}$; TE $306=5 \mathrm{~ms} ;$ flip angle $=30^{\circ} ; \mathrm{FOV}=6.4 \times 6.4 \mathrm{~mm}$; matrix $=64 \times 64 ;$ in-plane resolution $=100$ $307 \times 100 \mu \mathrm{m}^{2}$; slice thickness $=500 \mu \mathrm{m}$. A total TR for each image is $4 \mathrm{~s}$. The total acquisition 308 duration of each trial was 16 min. To measure the blood flow velocity, bipolar flow 309 encoding gradients were applied along the slice encoding direction. The slice position was anatomically identical with the slice position of the MGE imaging.

311 Data analysis and statistics

312 All data preprocessing and analysis were performed by using the software package, 313 Analysis of Functional Neurolmages (AFNI) (NIH, Bethesda). All relevant fMRI analysis 314 source codes can be downloaded from https://www.afni.nimh.nih.gov/afni/.

\section{Definition of the individual vessels}

316 The individual arteriole/venule voxels were defined by the signal intensity of the A-V $317 \mathrm{map}^{31}$. The arterioles are determined if the voxel intensities are higher than the mean 318 signal intensities plus two times the standard deviation of the local area in a $5 \times 5$ kernel.

319 The venules are determined if the voxel intensities are lower than the mean signal 320 intensities minus two times the standard deviation of the local area ${ }^{29-31}$. The locations of 321 individual arteriole/venule voxels defined in A-V map were used to extract the time 322 courses of BOLD/CBV-fMRI for individual vessels. 


\section{BOLD/CBV-fMRI and PC-MRI data analysis}

324 To register the evoked bSSFP-fMRI images and evoked PC-MRI images with the 2D anatomical A-V map, the tag-based registration method was applied. Twelve to fifteen tags were selected from the averaged bSSFP-fMRI images or the averaged PC-fMRI images to register those selected from A-V map. We used a 3dLocalstat AFNI function to normalize the signal intensity of the single-vessel maps. This process allowed us to plot the PC-based velocity values of individual vessel voxels to the normalized signal intensity of $\mathrm{A}-\mathrm{V}$ maps. For the evoked signals, the bSSFP-fMRI images and PC-MRI images were normalized by scaling the baseline to 100. Multiple trials of block-design time courses were averaged for each animal. No additional smoothing step was applied. The $\beta$-value was calculated to measure the amplitude of the fMRI responses at each TR. The voxelwise $\beta$-map was illustrated with the spatial pattern of the $f M R I$ responses corresponding to the different time points after the stimulus onset. After registration (tag-based registration) and region of interest extraction (3dLocalstat function, mask shown in

337 Figure 2B), we extracted the PC-based flow velocity values from individual vessel voxels, 338 which were identified based on the algorithm as described in the previous section.

339 The hemodynamic response function is based on the "block function" of 3dDeconvolve 340 module developed in AFNI. The HRF model is defined as follows:

$$
\mathrm{h}(\mathrm{t})=\int_{0}^{\min (t, L)} s^{4} e^{-s} /\left[4^{4} e^{-4}\right] \mathrm{ds}
$$

342 Gamma variate function $=\mathrm{s}^{4} \mathrm{e}^{-\mathrm{s}} / 4^{4} \mathrm{e}^{-4}$. $\mathrm{L}$ was the duration of the response. $\operatorname{BLOCK}(\mathrm{L}, 1)$

343 is a convolution of a square wave of duration $L$, makes a peak amplitude of block 344 response $=1$. 
For the group analysis, Student's t-test was performed, error bars are displayed as the

means \pm SEM. The $p$ values $<0.05$ were considered statistically significant. The sample

size of animal experiments is not previously estimated. No blinding and randomization

design was needed in this work.

\section{References}

350 1. Attwell, D. et al. Glial and neuronal control of brain blood flow. Nature 468, 232-243 (2010).

3512 2. Shih, A.Y. et al. Two-photon microscopy as a tool to study blood flow and neurovascular coupling in the rodent brain. J Cereb Blood Flow Metab 32, 1277-1309 (2012).

3. Kleinfeld, D., Mitra, P.P., Helmchen, F. \& Denk, W. Fluctuations and stimulus-induced changes in blood flow observed in individual capillaries in layers 2 through 4 of rat neocortex. Proceedings of the National Academy of Sciences of the United States of America 95, 15741-15746 (1998).

4. Jobsis, F.F. Noninvasive, infrared monitoring of cerebral and myocardial oxygen sufficiency and circulatory parameters. Science 198, 1264-1267 (1977).

5. Srinivasan, V.J. et al. Depth-resolved microscopy of cortical hemodynamics with optical coherence tomography. Optics letters 34, 3086-3088 (2009).

6. Yao, J., Maslov, K.I., Shi, Y., Taber, L.A. \& Wang, L.V. In vivo photoacoustic imaging of transverse blood flow by using Doppler broadening of bandwidth. Optics letters 35, 14191421 (2010).

7. Ances, B.M., Greenberg, J.H. \& Detre, J.A. Laser doppler imaging of activation-flow coupling in the rat somatosensory cortex. Neurolmage 10, 716-723 (1999).

8. Dunn, A.K., Bolay, H., Moskowitz, M.A. \& Boas, D.A. Dynamic imaging of cerebral blood flow using laser speckle. Journal of cerebral blood flow and metabolism : official journal of the International Society of Cerebral Blood Flow and Metabolism 21, 195-201 (2001).

9. Mace, E. et al. Functional ultrasound imaging of the brain. Nature methods 8, 662-664 (2011).

10. Sieu, L.A. et al. EEG and functional ultrasound imaging in mobile rats. Nature methods 12, 831-834 (2015).

11. Rungta, R.L., Osmanski, B.F., Boido, D., Tanter, M. \& Charpak, S. Light controls cerebral blood flow in naive animals. Nature communications 8, 14191 (2017).

12. Kwong, K.K. et al. Dynamic magnetic resonance imaging of human brain activity during primary sensory stimulation. Proceedings of the National Academy of Sciences of the United States of America 89, 5675-5679 (1992).

13. Detre, J.A., Leigh, J.S., Williams, D.S. \& Koretsky, A.P. Perfusion imaging. Magnetic resonance in medicine : official journal of the Society of Magnetic Resonance in Medicine / Society of Magnetic Resonance in Medicine 23, 37-45 (1992).

14. Williams, D.S., Detre, J.A., Leigh, J.S. \& Koretsky, A.P. Magnetic resonance imaging of perfusion using spin inversion of arterial water. Proceedings of the National Academy of Sciences of the United States of America 89, 212-216 (1992).

15. Ogawa, S., Lee, T.M., Kay, A.R. \& Tank, D.W. Brain magnetic resonance imaging with contrast dependent on blood oxygenation. Proceedings of the National Academy of Sciences of the United States of America 87, 9868-9872 (1990).

16. Ogawa, S. et al. Intrinsic signal changes accompanying sensory stimulation: functional brain mapping with magnetic resonance imaging. Proceedings of the National Academy of Sciences of the United States of America 89, 5951-5955 (1992). 
390

391

392

393

394

395

396

397

398

399

400

401

402

403

404

405

406

407

408

409

410

411

412

413

414

415

416

417

418

419

420

421

422

423

424

425

426

427

428

429

430

431

432

433

434

435

436

437

438

17. Belliveau, J.W. et al. Functional mapping of the human visual cortex by magnetic resonance imaging. Science 254, 716-719 (1991).

18. Moran, P.R. A flow velocity zeugmatographic interlace for NMR imaging in humans. Magnetic resonance imaging 1, 197-203 (1982).

19. Bryant, D.J., Payne, J.A., Firmin, D.N. \& Longmore, D.B. Measurement of flow with NMR imaging using a gradient pulse and phase difference technique. Journal of computer assisted tomography 8, 588-593 (1984).

20. Detre, J.A. \& Wang, J. Technical aspects and utility of fMRI using BOLD and ASL. Clin Neurophysiol 113, 621-634 (2002).

21. Zong, X. \& Lin, W. Quantitative phase contrast MRI of penetrating arteries in centrum semiovale at 7T. Neurolmage 195, 463-474 (2019).

22. Bouvy, W.H. et al. Assessment of blood flow velocity and pulsatility in cerebral perforating arteries with 7-T quantitative flow MRI. NMR in biomedicine 29, 1295-1304 (2016).

23. Kang, C.K. et al. Velocity measurement of microvessels using phase-contrast magnetic resonance angiography at 7 Tesla MRI. Magnetic resonance in medicine : official journal of the Society of Magnetic Resonance in Medicine / Society of Magnetic Resonance in Medicine 75, 1640-1646 (2016).

24. Schnerr, R.S. et al. Pulsatility of Lenticulostriate Arteries Assessed by 7 Tesla Flow MRIMeasurement, Reproducibility, and Applicability to Aging Effect. Front Physiol 8, 961 (2017).

25. Wei, Z. et al. Optimization of phase-contrast MRI for the estimation of global cerebral blood flow of mice at 11.7T. Magnetic resonance in medicine : official journal of the Society of Magnetic Resonance in Medicine / Society of Magnetic Resonance in Medicine 81, 25662575 (2019).

26. Dolui, S. et al. Comparison of non-invasive MRI measurements of cerebral blood flow in a large multisite cohort. Journal of cerebral blood flow and metabolism : official journal of the International Society of Cerebral Blood Flow and Metabolism 36, 1244-1256 (2016).

27. Geurts, L.J. et al. Vascular reactivity in small cerebral perforating arteries with 7T phase contrast MRI - A proof of concept study. Neurolmage 172, 470-477 (2018).

28. Hoogeveen, R.M., Bakker, C.J. \& Viergever, M.A. MR phase-contrast flow measurement with limited spatial resolution in small vessels: value of model-based image analysis. Magnetic resonance in medicine : official journal of the Society of Magnetic Resonance in Medicine / Society of Magnetic Resonance in Medicine 41, 520-528 (1999).

29. He, Y. et al. Ultra-Slow Single-Vessel BOLD and CBV-Based fMRI Spatiotemporal Dynamics and Their Correlation with Neuronal Intracellular Calcium Signals. Neuron 97, 925-939 e925 (2018).

30. Chen, X. et al. Mapping optogenetically-driven single-vessel fMRI with concurrent neuronal calcium recordings in the rat hippocampus. Nature communications 10, 5239 (2019).

31. Yu, X. et al. Sensory and optogenetically driven single-vessel fMRI. Nature methods 13, 337-340 (2016).

32. Walker, P.G. et al. Semiautomated method for noise reduction and background phase error correction in MR phase velocity data. Journal of magnetic resonance imaging : JMRI 3, 521-530 (1993).

33. Peeters, J.M., Bos, C. \& Bakker, C.J. Analysis and correction of gradient nonlinearity and B0 inhomogeneity related scaling errors in two-dimensional phase contrast flow measurements. Magnetic resonance in medicine : official journal of the Society of Magnetic Resonance in Medicine / Society of Magnetic Resonance in Medicine 53, 126133 (2005). 
34. Bernstein, M.A. et al. Concomitant gradient terms in phase contrast MR: analysis and correction. Magnetic resonance in medicine : official journal of the Society of Magnetic

35. Schaffer, C.B. et al. Two-photon imaging of cortical surface microvessels reveals a robust redistribution in blood flow after vascular occlusion. PLoS Biol 4, e22 (2006).

36. Silva, A.C., Koretsky, A.P. \& Duyn, J.H. Functional MRI impulse response for BOLD and CBV contrast in rat somatosensory cortex. Magnet Reson Med 57, 1110-1118 (2007).

37. Drew, P.J., Shih, A.Y. \& Kleinfeld, D. Fluctuating and sensory-induced vasodynamics in rodent cortex extend arteriole capacity. Proc. Natl. Acad. Sci. USA 108, 8473-8478 (2011).

38. Koretsky, A.P. Early development of arterial spin labeling to measure regional brain blood flow by MRI. Neurolmage 62, 602-607 (2012).

39. Wang, J., Fernandez-Seara, M.A., Wang, S. \& St Lawrence, K.S. When perfusion meets diffusion: in vivo measurement of water permeability in human brain. Journal of cerebral blood flow and metabolism : official journal of the International Society of Cerebral Blood Flow and Metabolism 27, 839-849 (2007).

40. Hutchinson, E.B., Stefanovic, B., Koretsky, A.P. \& Silva, A.C. Spatial flow-volume dissociation of the cerebral microcirculatory response to mild hypercapnia. Neurolmage $32,520-530$ (2006). 
A

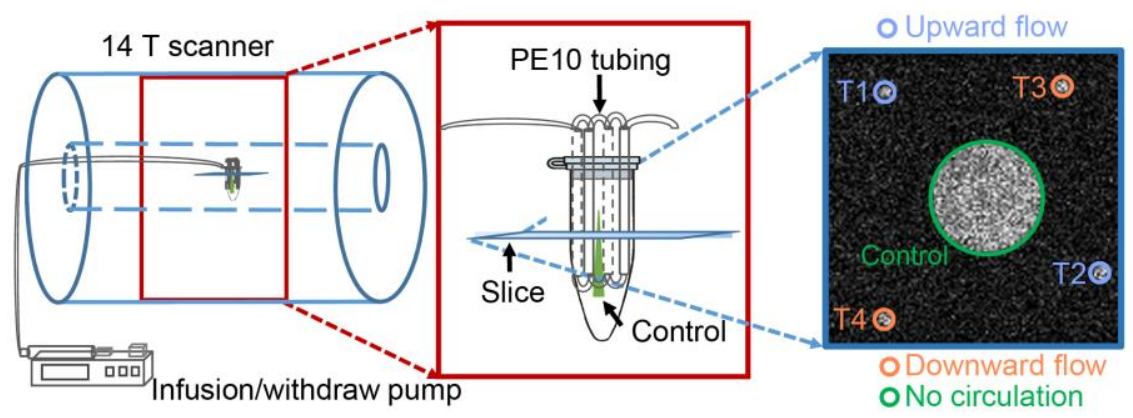

B

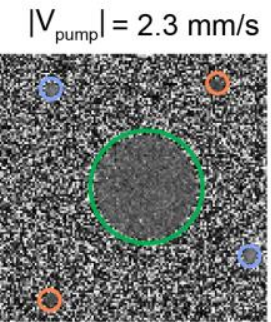

$4.5 \mathrm{~mm} / \mathrm{s}$

$6.8 \mathrm{~mm} / \mathrm{s}$
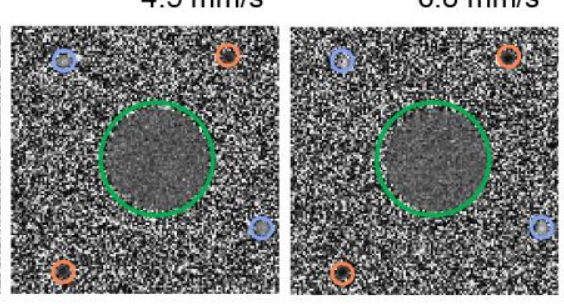

$9.0 \mathrm{~mm} / \mathrm{s}$
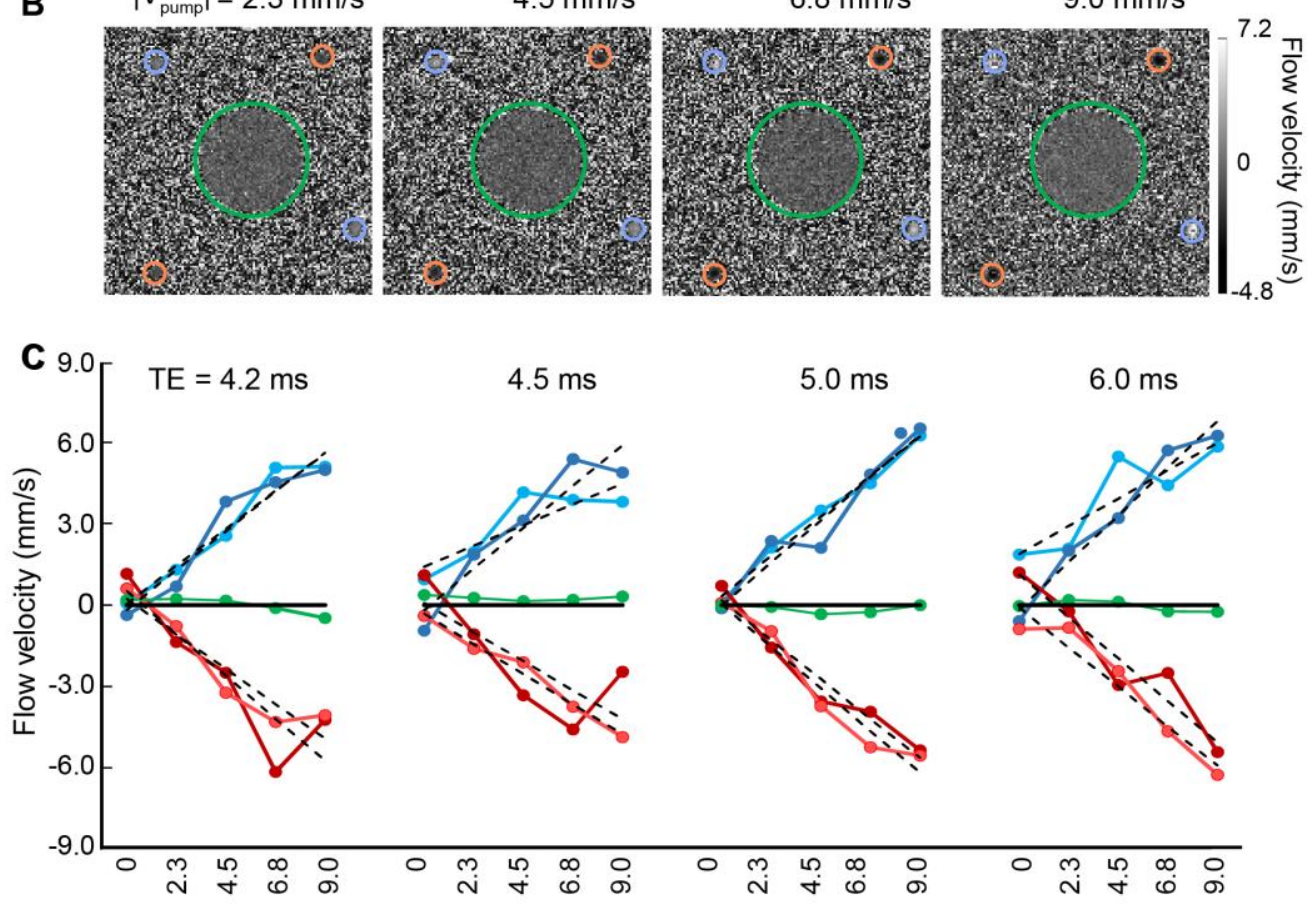

$6.0 \mathrm{~ms}$

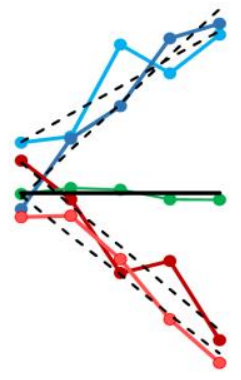

Figure 1. In vitro flow velocity measurements with phase-contrast (PC)-MRI. A. Schematic drawing of the phantom experimental flow chamber in the 14.1 $\mathrm{T}$ scanner. An expanded image (red box) shows the circulatory system constructed of capillary tubes. A representative fast low angle shot (FLASH) MRI image (blue box), $500 \mu \mathrm{m}$ in thickness, shows the capillary positions.

478 regions of interest (ROIs) T1 and T2, contoured in purple, indicate the upward flow. ROls T3 and $479 \mathrm{~T} 4$, in orange contour, indicate the downward flow. The green contour indicates the stagnant fluid. 480 B. Representative images with different flow velocity in the capillaries T1 to T4 in panel A. (echo 481 time) $T E=5.0 \mathrm{~ms}$ for all panels. C. The plot of flow velocity estimates from the five ROls with 482 different TEs, as marked, and different pump rates, as indicated and marked in panel B. The 483 dotted lines correspond to a linear fitting for velocity measurements of different ROls. 

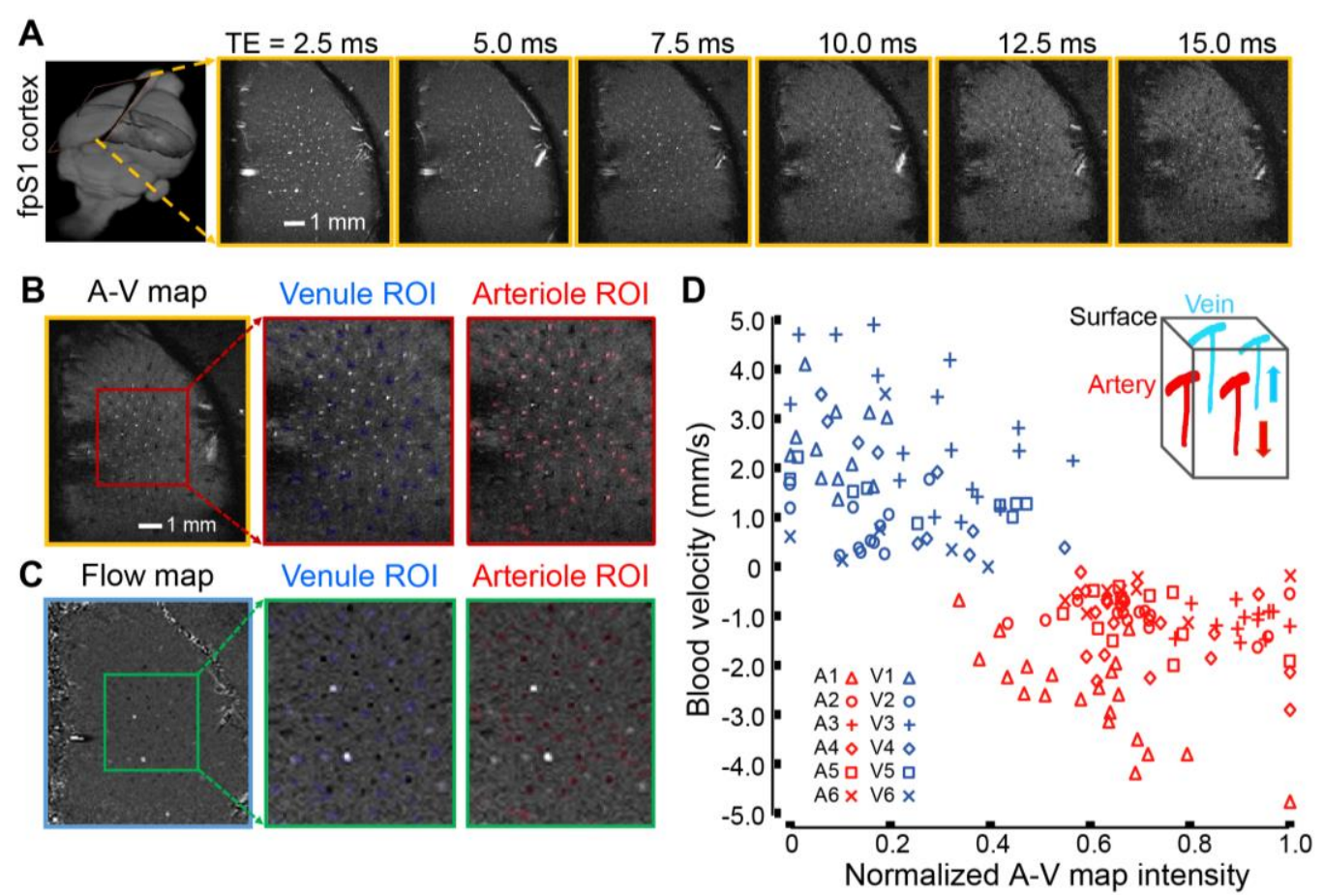

487 Figure 2. Single-vessel flow velocity measurement. A. Representative 2D multi-gradient echo 488 (MGE) slices (yellow boxes) from a deep layer of the primary forepaw somatosensory cortex (first 489 frame) at different TEs, as indicated. B. The 2D arteriole-venule (A-V) map (yellow box) derived 490 from the images with different TEs in panel $A$, arterioles and venules appear as bright and dark 491 voxels, respectively. The expanded views (red boxes) show individual venules, i.e., black voxels 492 marked in blue, and arterioles, i.e., white voxels marked in red. C. The vectorized flow velocity 493 map (blue box) from the same 2D MGE slice in panel B. The expanded views (green boxes) show 494 the individual venules, i.e., white dots with positive velocity, and arterioles, i.e., black dots with 495 negative velocity. Note that 2 bright dots are caused by the "over-flowed" velocity beyond the 496 maximal velocity, i.e., the velocity encoding (Venc) parameter, defined in the PC-MRI sequence, 497 which could be not correctly estimated. D. Scatter plot of the flow velocities from individual 498 arterioles and venules as the function of the normalized signal intensities of each vessel in the A$499 \quad V$ map of panel $B$, data from 6 rats as indicated. Insert shows the blood flow direction of arterioles 500 and venules in the forepaw somatosensory cortical region. 

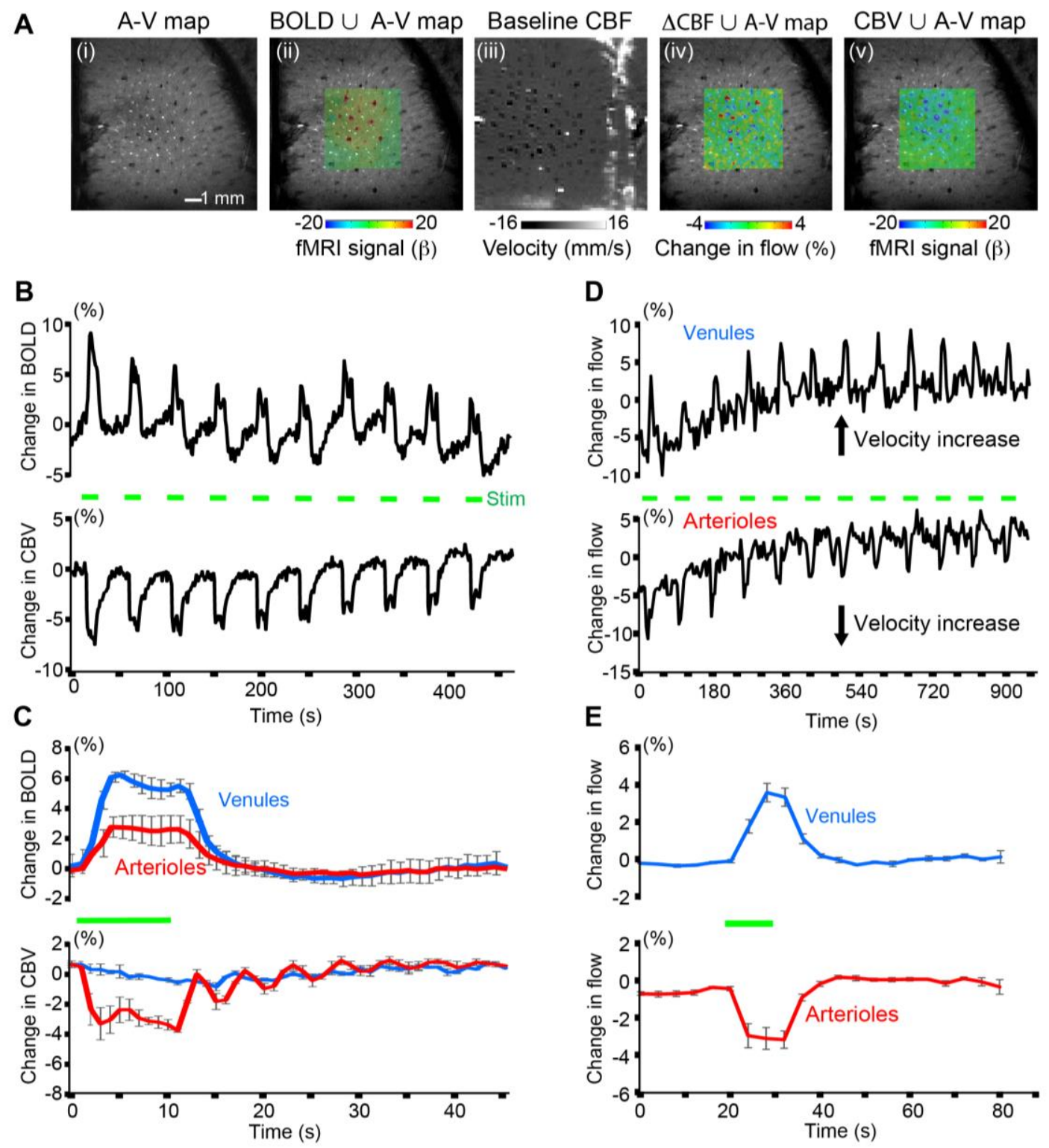

Figure 3. Maps of task-related hemodynamic signals with BOLD, CBV, and CBF-fMRI. A.

Different MRI measurement strategies on the same 2D slice. From left to right: (i) The singlevessel A-V map acquired with the MGE method; (ii) The evoked balanced steady-state free precession (bSSFP)-based BOLD-fMRI signal, within the green subregion, on top of the A-V map; (iii) the PC-MRI map of baseline CBF; (iv) the change in CBF on top of the A-V map with an increased flow velocity corresponding to brighter voxels for venules and darker voxels for arterioles; and ( $v$ ) the evoked bSSFP-based CBV-fMRI signal on top of the A-V map. B. The time courses of the evoked bSSFP-BOLD and CBV-fMRI with the block-design paradigm from the venules and arterioles shown in panel A. Forepaw stimulation pulse of $330 \mu \mathrm{s}$ in width and $1 \mathrm{~mA}$ 
512 the evoked BOLD and CBV signals from the venule and arteriole ROls from different rats (mean

$513 \pm$ SEM, the green bar shows stimulation duration). The peak BOLD values of venule are

514 significantly higher than those of arteriole ( 5 rats, $p=0.009$ ), while the peak CBV values of

515 arteriole are significantly higher than those of venule ( 3 rats, $p=0.028$ ). D. The time courses of

516 the evoked CBF changes from the arteriole and venule ROls show increased velocity from both

517 arterioles and venules with the block-design, $10 \mathrm{~s}$ duration stimulation paradigm. E. The

518 averaged time courses of the evoked CBF changes show the velocity increase from both arteriole

519 and venule ROls with the block-design stimulation paradigm from 4 rats (mean \pm SEM, the green

520 bar shows stimulation duration). 
A

A

Anesthesia
pump

525

526

527

528

529
14.1 T scanner
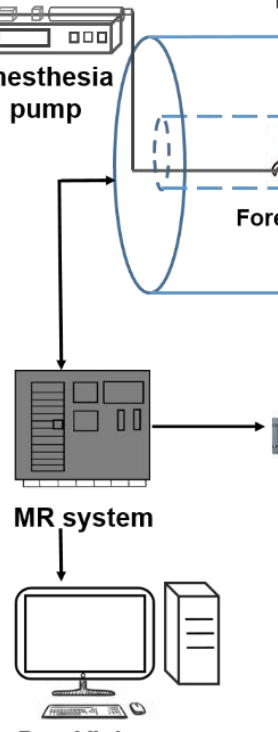

ParaVision
B

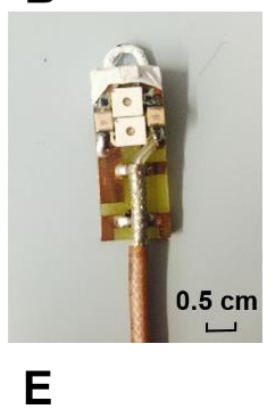

C
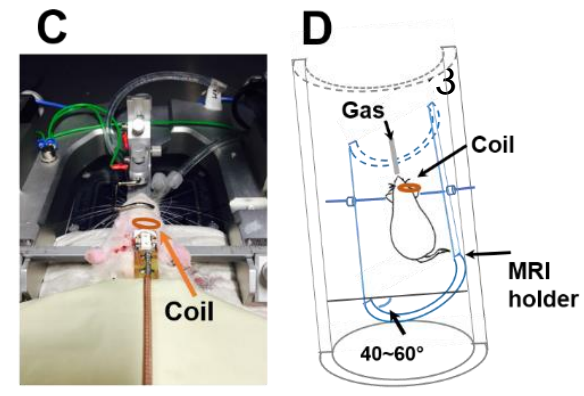

E
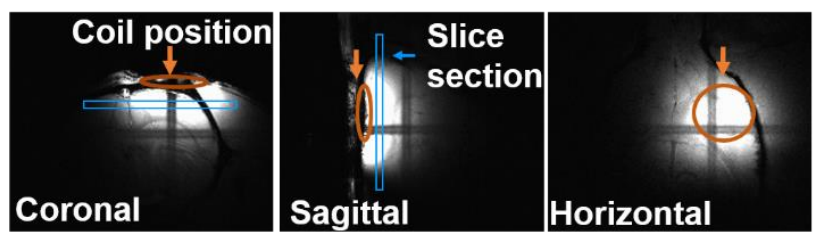

Figure S1. The preparation of in vivo experiment for the PC-MRI in 14.1 T.

A. The flow chart of the in vivo experiment in the $14.1 \mathrm{~T}$ scanner.

B. Photograph of the custom-made transceiver surface RF coil.

C. Photograph of the coil position: the coil is glued to the rat skull.

D. The schematic drawing of the rat position inside the MRI holder.

E. Representative images from different views of the FLASH MRI show the ideal coil position. 
A

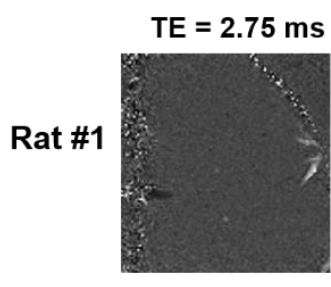

B

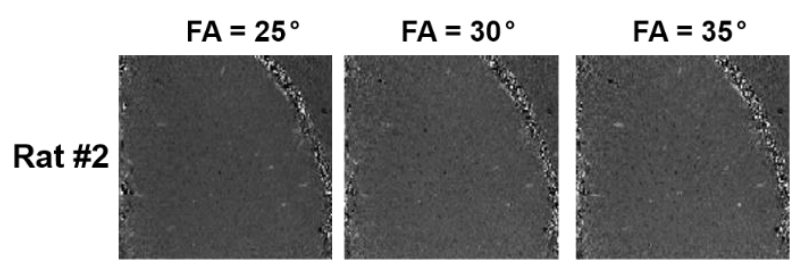

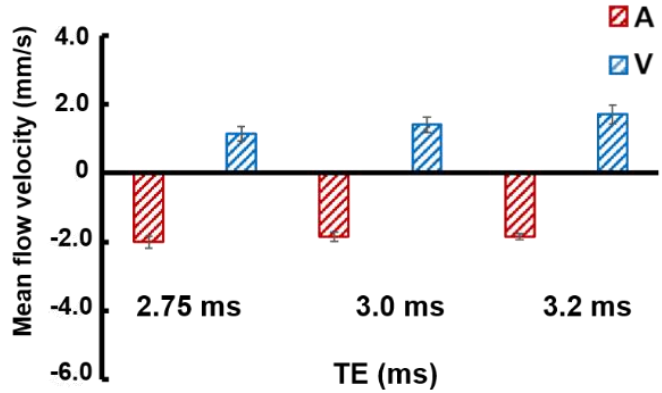

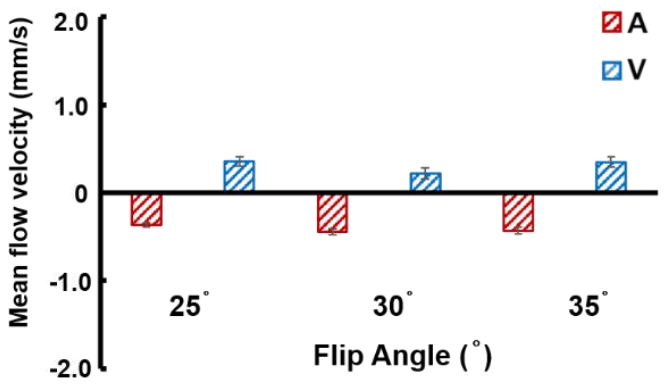

Figure S2. Phase images from the different representative rats with different TEs and flip angles.

A. Phase images from a representative rat with different TEs, i.e., 2.75, 3.0, and $3.2 \mathrm{~ms}$. The right panel shows the mean blood flow velocity (mean \pm SEM) from left images with $\mathrm{N}_{\text {Arteriole }}=48$ and $\mathrm{N}_{\text {venule }}=22$.

B. Phase images from a representative rat with different flip angles, i.e., $25^{\circ}, 30^{\circ}$, and $35^{\circ}$. The right panel shows the mean blood flow velocity from left images with $N_{\text {Arteriole }}=38$ and $N_{\text {venule }}=14$. 
A

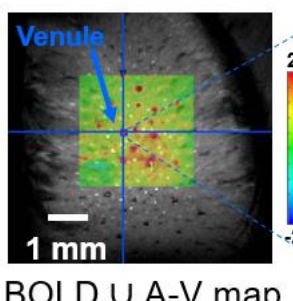

B

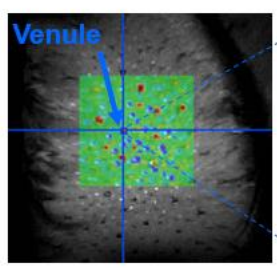

$\triangle \mathrm{CBF} \cup \mathrm{A}-\mathrm{V}$ map

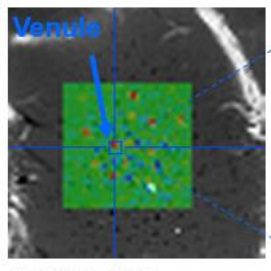

$\triangle \mathrm{CBF} \cup$ flow map
$3 \times 3$ voxel-wise time course
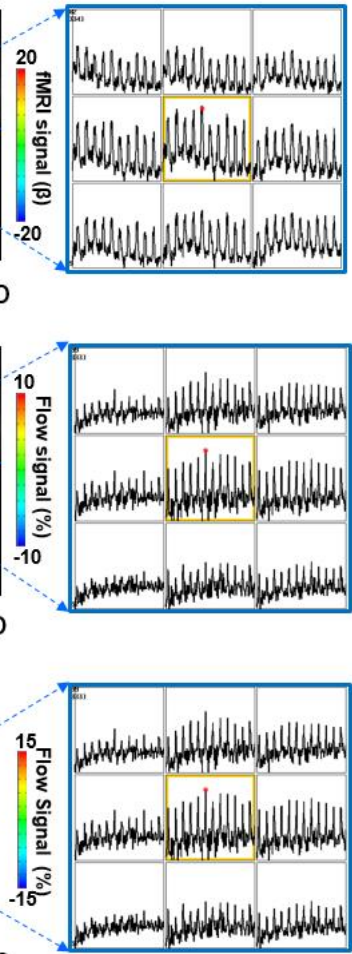

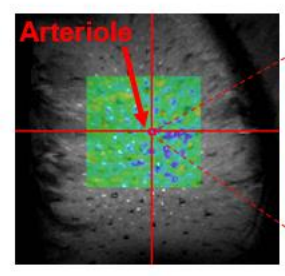

$\mathrm{CBV} \cup \mathrm{A}-\mathrm{V}$ map

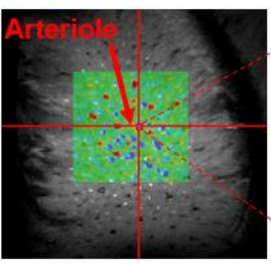

$\triangle \mathrm{CBF} \cup \mathrm{A}-\mathrm{V}$ map
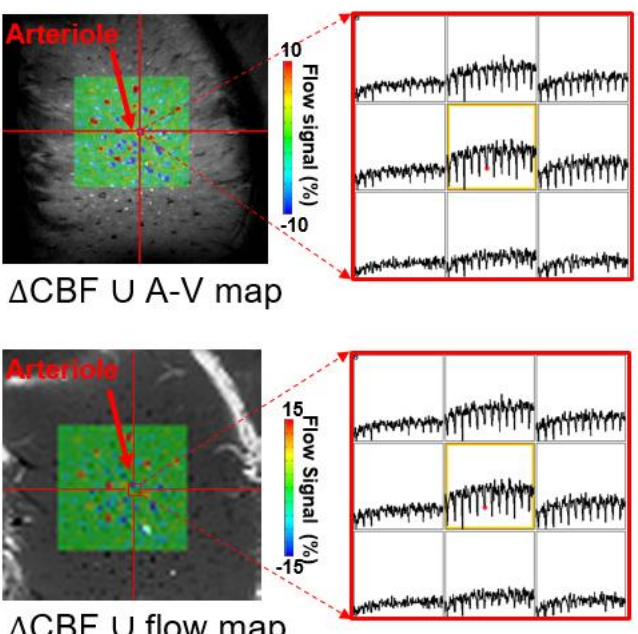

Figure S3. The bSSFP-based single-vessel BOLD/CBV-fMRI and the PC-MRI based singlevessel dynamic flow measurement from a representative rat.

A. The evoked bSSFP-based BOLD- (left) and CBV- (right) fMRI maps overlaid on the same A-V map from a representative rat, with the voxel-wise time courses from the ROls of individual venule (blue arrow) and arteriole (red arrow) (10 s on and $35 \mathrm{~s}$ off for 10 epochs plotted in a $3 \times 3$ matrix). B. The evoked CBF maps overlaid on both A-V map (upper panel) and PC-based flow map (lower panel) from the same representative rat. The voxel-wise time courses of CBF changes from the same ROls of individual venule (blue arrow) and arteriole (red arrow) (10 s on and $50 \mathrm{~s}$ off for 12 epochs plotted in a $3 \times 3$ matrix). 


\section{Supplementary Movie Legends}

Movie 1. The bSSFP-based single-vessel BOLD/CBV-fMRI and the PC-MRI based singlevessel dynamic flow measurement in the rat cortex.

The upper panel shows the evoked bSSFP-based BOLD- (left) and CBV- (right) fMRI maps overlaid on the 2D A-V map with $50 \times 50 \mu \mathrm{m}^{2}$ in-plane and the time course from a single voxel located at a representative venule (blue arrow) and arteriole (red arrow) (10 $\mathrm{s}$ on and $35 \mathrm{~s}$ off for a total of $45 \mathrm{~s}$ time window with $\mathrm{TR}=1 \mathrm{~s}$ ). The middle panel shows the evoked CBF maps overlaid on the same 2D A-V map from the same individual venule (left) and arteriole (right), of which the CBF-fMRI maps were registered to match the $2 \mathrm{D} \mathrm{A-V}$ map at the $50 \times 50 \mu \mathrm{m}^{2}$ resolution, as well as the time courses of the PC-based flow velocity dynamic changes from same voxels identified as the upper panel ( $10 \mathrm{~s}$ on and $50 \mathrm{~s}$ off for a total of $60 \mathrm{~s}$ time window with $\mathrm{TR}=4 \mathrm{~s}$ ). The lower panel shows the evoked CBF maps overlaid on the PC-based flow map with $100 \times 100 \mu \mathrm{m}^{2}$ resolution and the time course from the voxels located at the representative venule (left) and arteriole (right). Note that the venule is a bright voxel and arteriole is a dark voxel in the flow map, which is opposite to the A-V map and also the slightly different time course due to the altered spatial resolution.
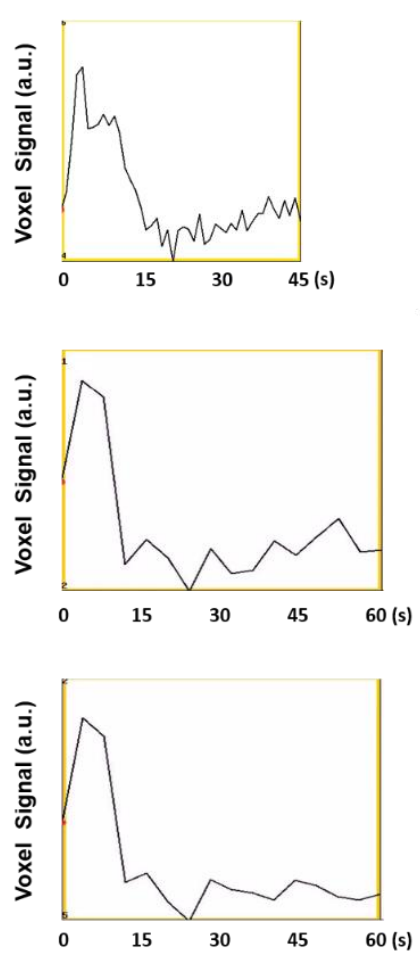
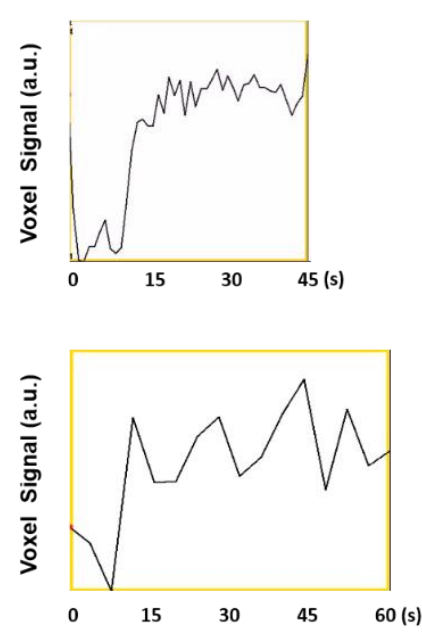
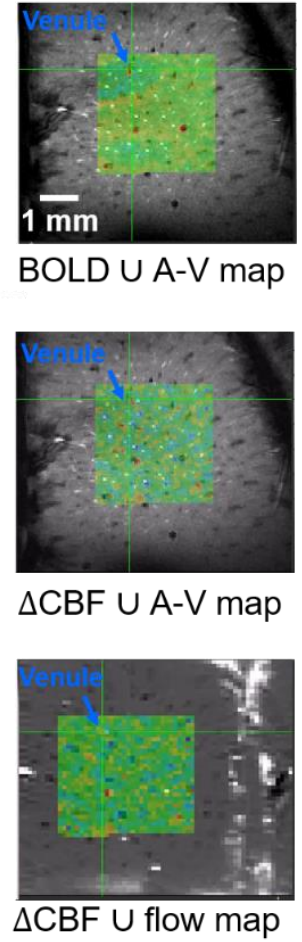

BOLD $\cup$ A-V map

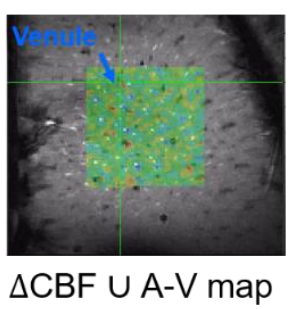

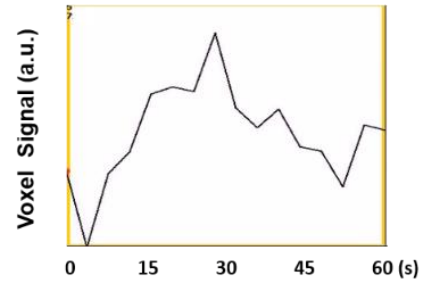

$\triangle \mathrm{CBF} \cup \mathrm{A}-\mathrm{V}$ map
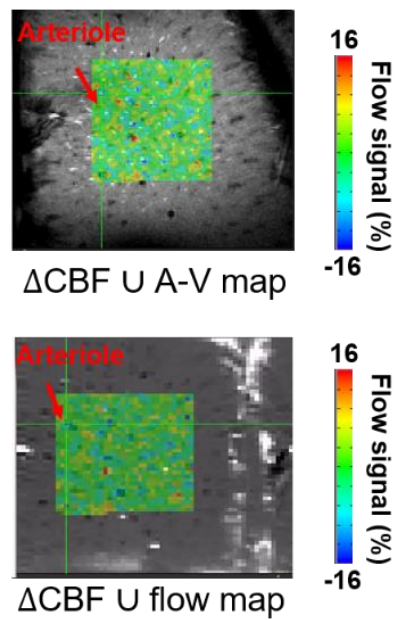
A
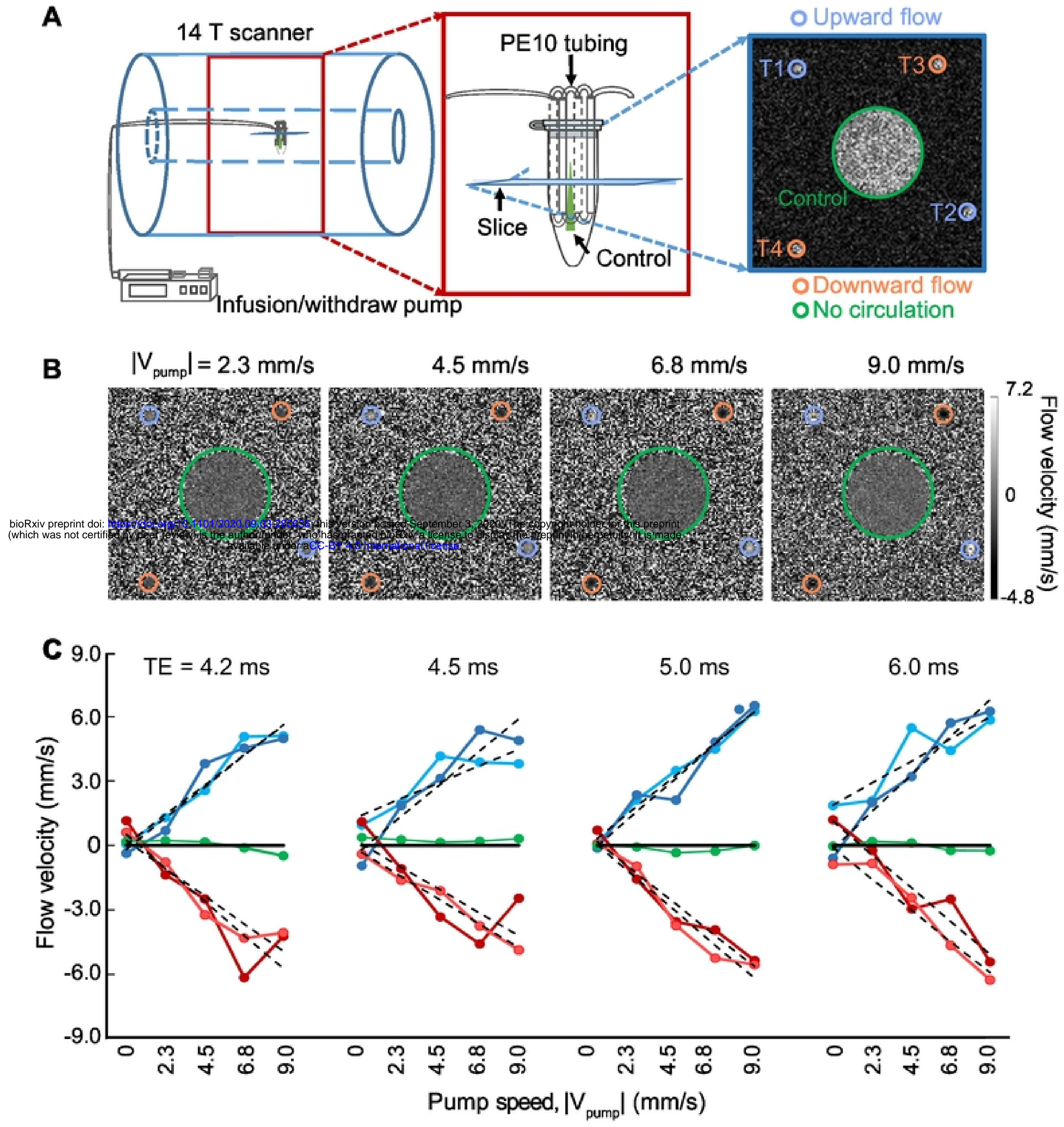

Figure 1. Chen, Jiang, Choi, Pohmann, Scheffler, Kleinfeld and Yu

Figure 1 

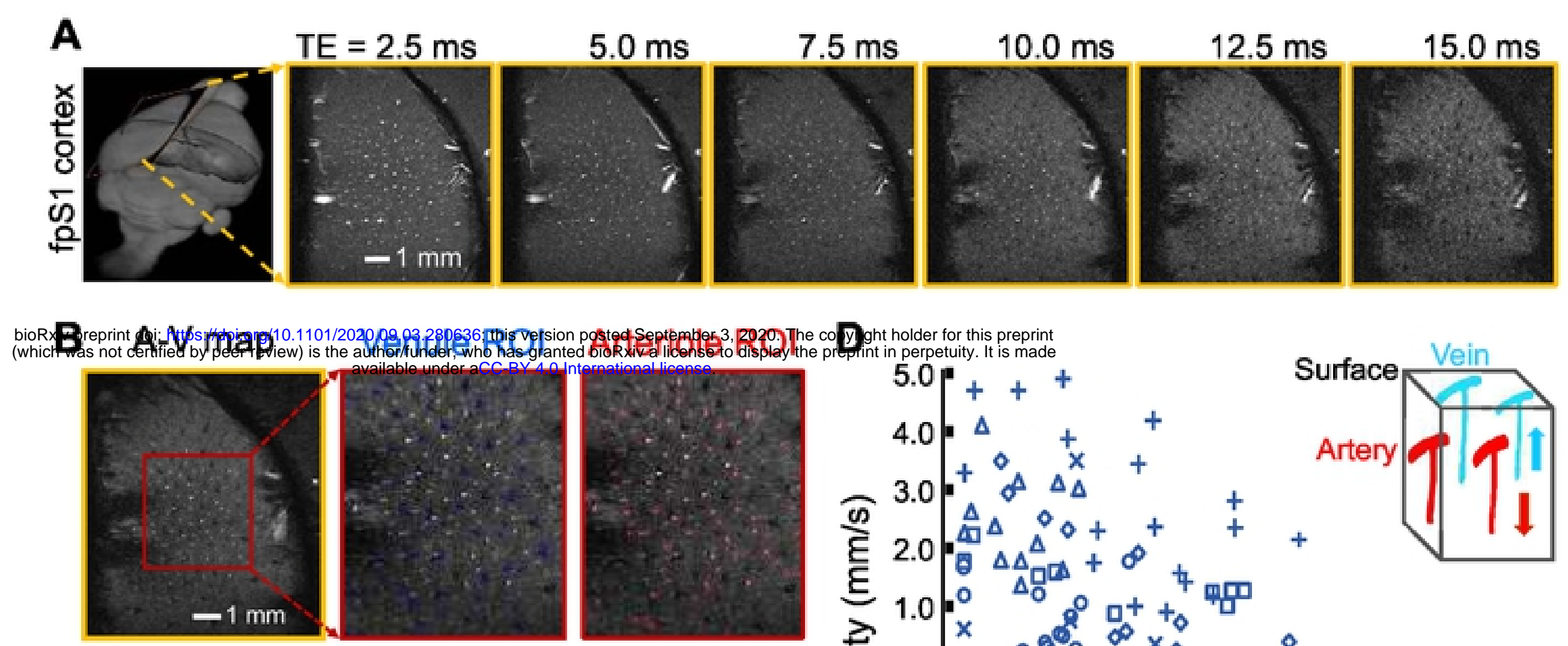

ght holder for this preprint

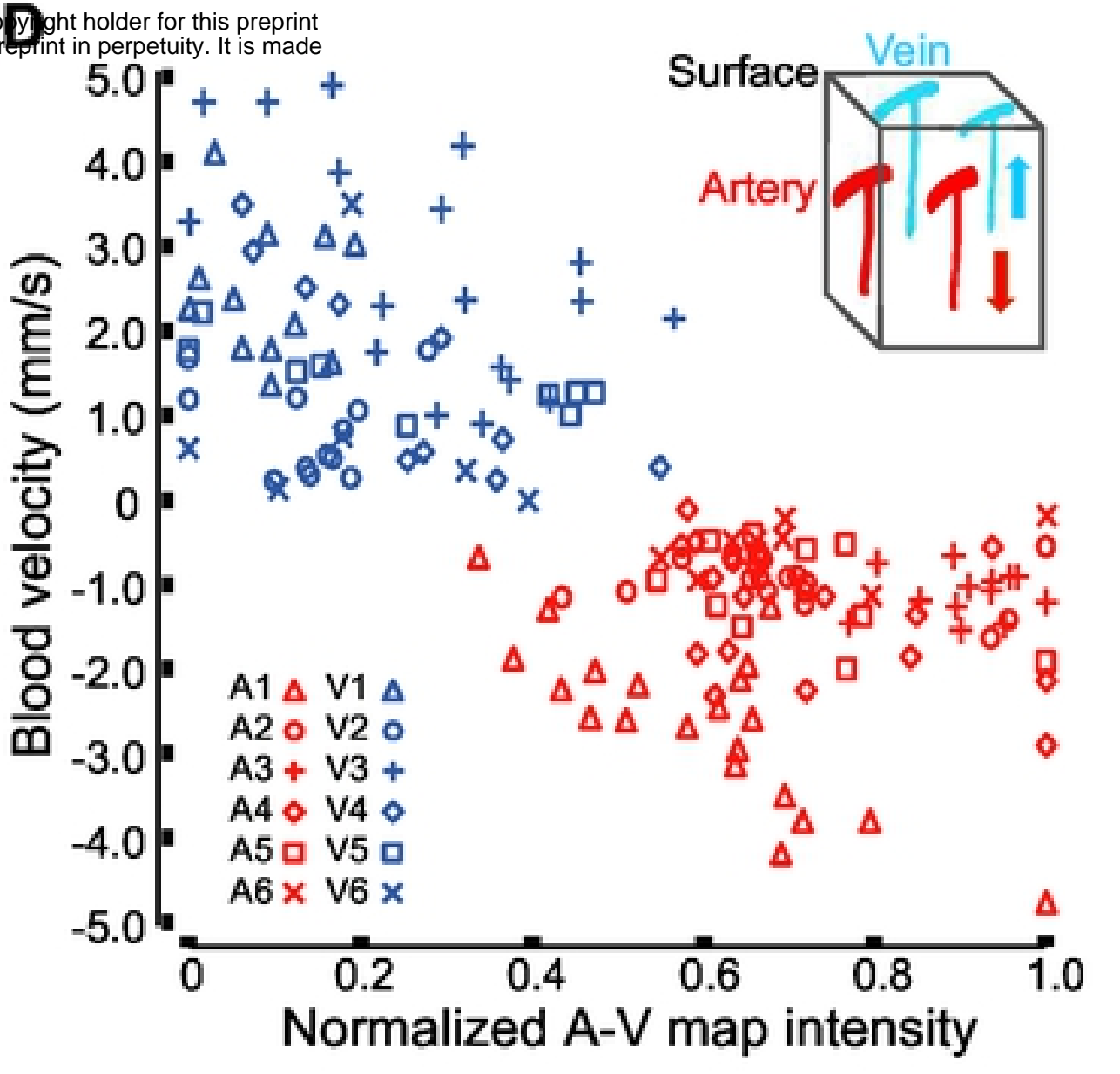

Figure 2. Chen, Jiang, Choi, Pohmann, Scheffler, Kleinfeld and Yu

Figure 2 


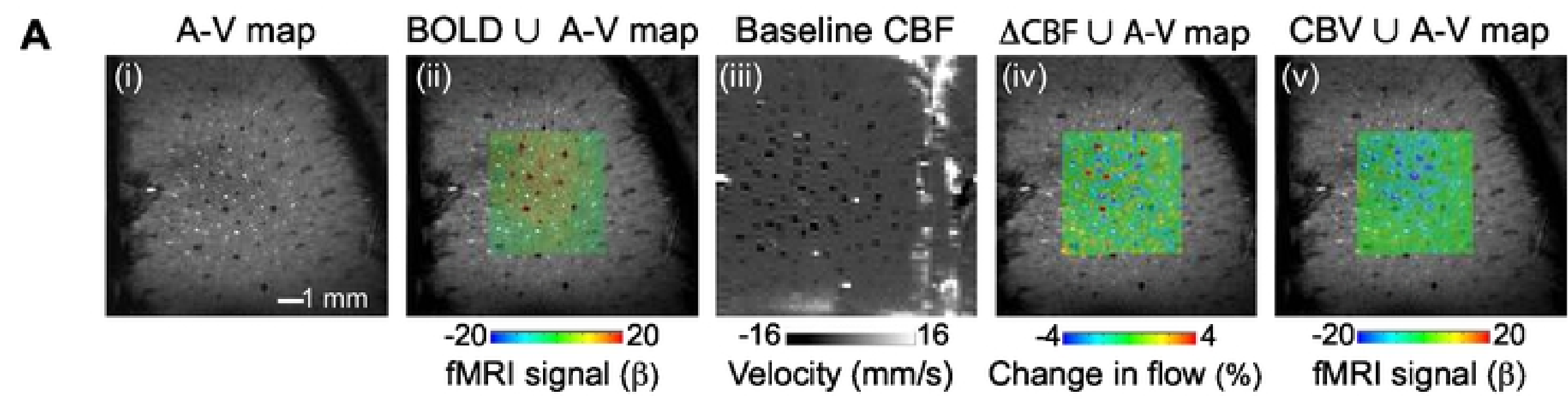

B

D

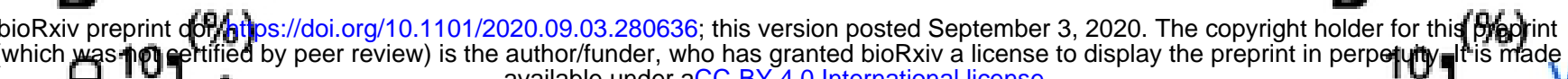
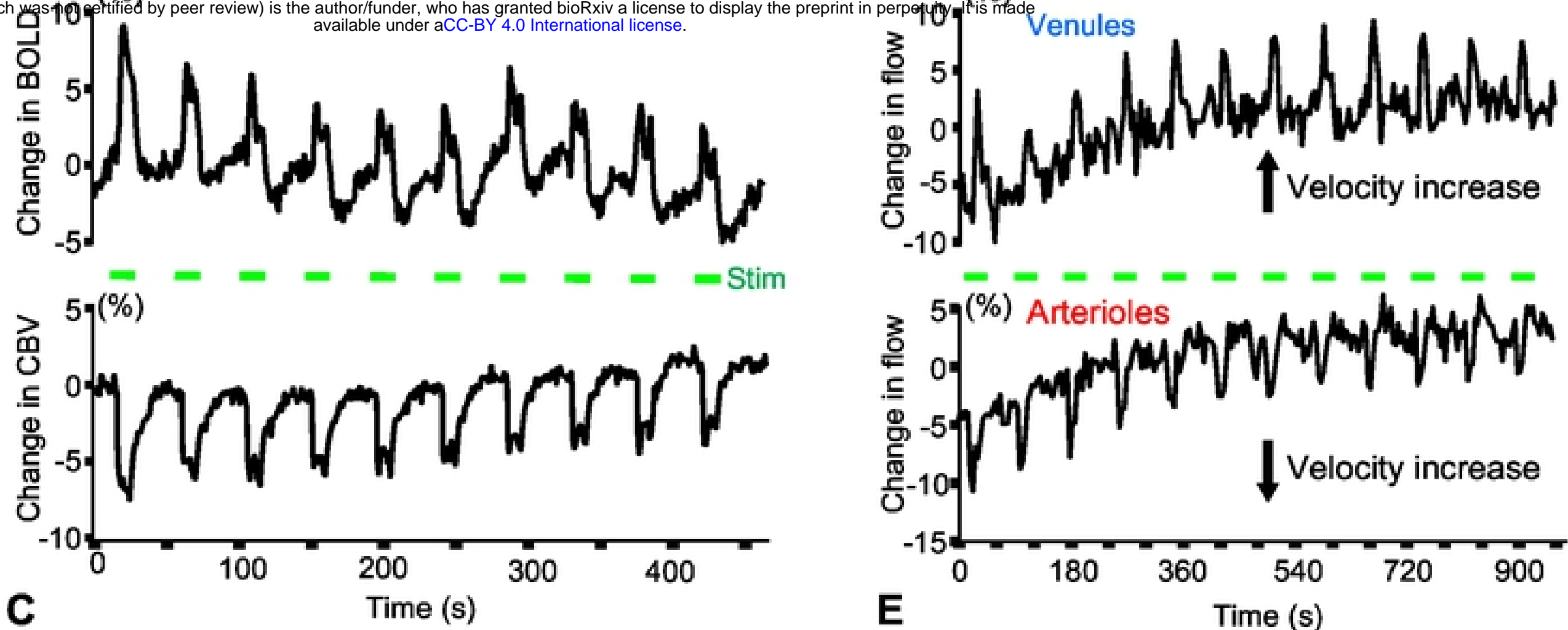

C

Time (s)

E
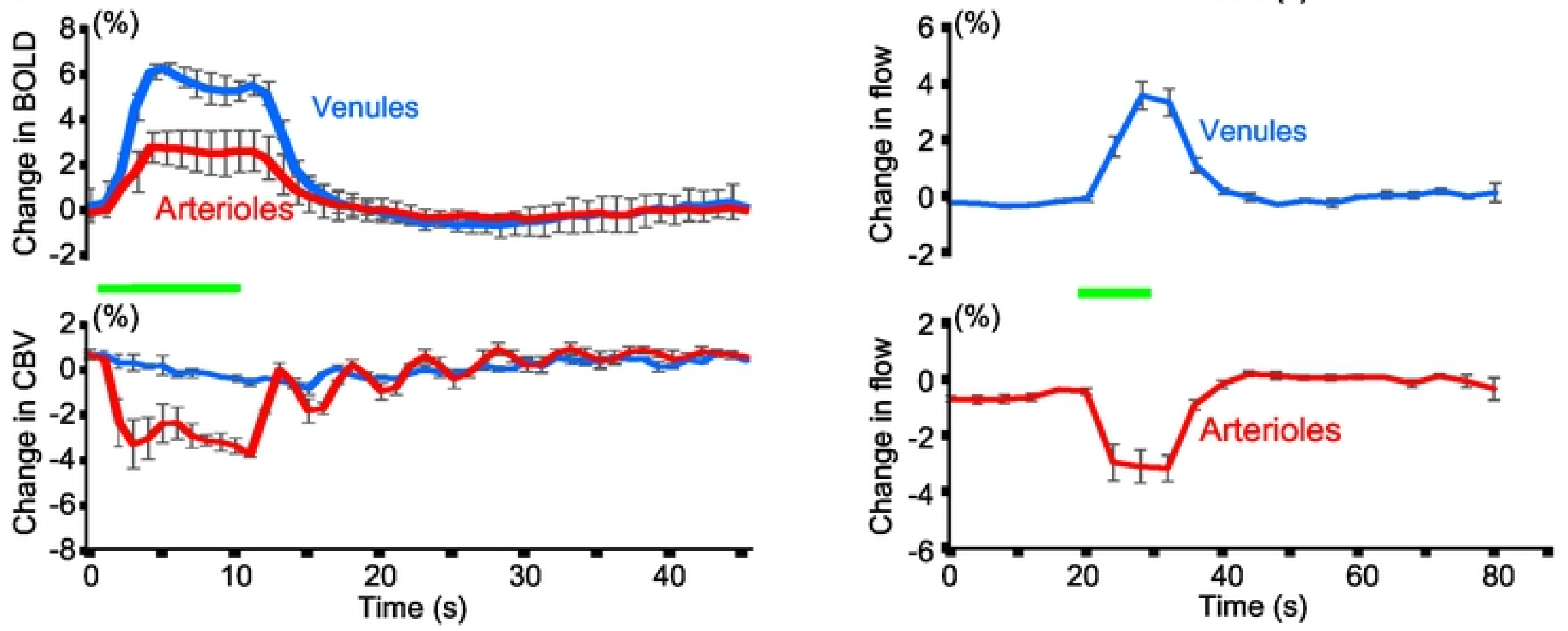

Figure 3. Chen, Jiang, Choi, Pohmann, Scheffler, Kleinfeld and Yu

Figure 3 